PROCEEDINGS OF THE

AMERICAN MATHEMATICAL SOCIETY

Volume 137, Number 6, June 2009, Pages 1971-1976

S 0002-9939(09)09683-X

Article electronically published on January 27, 2009

\title{
BOUNDARY POINTS AS LIMIT FUNCTIONS OF ITERATED HOLOMORPHIC FUNCTION SYSTEMS
}

\author{
KOUROSH TAVAKOLI \\ (Communicated by Jane M. Hawkins) \\ This paper is dedicated to my mother.
}

\begin{abstract}
We show that if $c$ is a boundary point of a non-relatively compact subdomain of the unit disk, there always exists an iterated holomorphic function system with the constant $c$ as a limit function.
\end{abstract}

\section{INTRODUCTION}

Let $\Omega$ be a hyperbolic domain in the plane. Suppose $\left\{f_{n}\right\}$ is a sequence of holomorphic maps from $\Omega$ into a subdomain $X \subset \Omega$.

Consider the compositions

$$
F_{n}=f_{1} \circ f_{2} \circ \ldots f_{n-1} \circ f_{n} .
$$

The sequence $\left\{F_{n}\right\}$ is called an iterated function system. $\left\{F_{n}\right\}$ is a family of holomorphic maps from $\Omega$ into the subdomain $X \subset \Omega$. Since $X$ is a hyperbolic domain, by Montel's theorem, the iterated function system $\left\{F_{n}\right\}$ is a normal family. Therefore, there is a subsequence of the sequence $\left\{F_{n}\right\}$ which converges locally uniformly to a holomorphic map $F$ or to infinity. The limit functions are either open maps from $\Omega$ into $X$ or constants in $\bar{X}$.

Definition 1.1. A subdomain $X$ of a domain $\Omega$ is called relatively compact in $\Omega$ if there exists a compact set $K$ such that $X \subset K \subset \Omega$. Otherwise, $X$ is called non-relatively compact in $\Omega$.

Suppose $\Omega$ is the unit disk $\Delta$. Lorentzen [7] and Gill 3 showed that if $X$ is relatively compact in $\Delta$, then every iterated function system converges to a unique constant which is inside $X$ not on its boundary. Therefore, non-relative compactness of $X$ in $\Delta$ is a necessary condition in order to have a boundary point of $X$ as a limit function of an iterated function system. In this paper, we want to show that for any point $c$ on the boundary of $X$, the non-relative compactness of $X$ in $\Delta$ is a sufficient condition for the existence of an iterated function system that has the constant $c$ as a limit function.

Received by the editors October 31, 2007.

2000 Mathematics Subject Classification. Primary 30D05; Secondary 30C35, 30C70, 30C75, $37 \mathrm{~F} 10$.

Key words and phrases. Iterated function system, non-relatively compact, holomorphic map. 
This paper is organized as follows. In section 2, the key facts are stated and proved. Section 3 is devoted to the statement and the proof of the main theorem and an interesting corollary.

\section{KEY FACTS}

First we state a lemma of Keen and Lakic which is used later in this section to prove the key theorem. We will bring the proof of the lemma, and in the remark following the proof we explain how the lemma can be used in proving the key theorem.

Lemma 2.1 (Keen-Lakic). Suppose $X$ is a non-relatively compact subdomain of the unit disk $\Delta$. Let $a_{1}, \ldots, a_{n}$ be $n$ distinct points in $\Delta \backslash\{0\}$. Then there exist a holomorphic function $f: \Delta \rightarrow \Delta$ and points $x_{1}, \ldots, x_{n} \in X$ such that $f\left(x_{i}\right)=$ $a_{i} / x_{i}$, for all $i=1, \ldots, n$.

Proof. The proof will make use of the automorphisms

$$
A(a, z)=\frac{z-a}{1-\bar{a} z} .
$$

Step 1 . Since $X$ is not relatively compact we may choose an $x_{1} \in X$ such that $\left|x_{1}\right|>\left|a_{1}\right|$. Let $g_{1}(z)$ be a self-map of the unit disk to be determined. Define

$$
f(z)=\frac{A\left(x_{1}, z\right) g_{1}\left(A\left(x_{1}, z\right)\right)+\frac{a_{1}}{x_{1}}}{1+\frac{\bar{a}_{1}}{\bar{x}_{1}} A\left(x_{1}, z\right) g_{1}\left(A\left(x_{1}, z\right)\right)} .
$$

It follows that $f\left(x_{1}\right)=a_{1} / x_{1}$ as required. Because we want to work inductively we rewrite this definition implicitly as follows:

$$
A\left(x_{1}, z\right) g_{1}\left(A\left(x_{1}, z\right)\right)=A\left(\frac{a_{1}}{x_{1}}, f(z)\right) .
$$

If $n=1$ we set $g_{1}(z) \equiv 0$ and we are done. From now on we assume that $n>1$ and that we have chosen $x_{1}$.

Step 2. Before we proceed, we set up some further notation:

For $1 \leq j \leq k \leq n$, set $a_{j k}=A\left(x_{j}, x_{k}\right)$. Next, for $k=2, \ldots, n$, set

$$
b_{1 k}=A\left(\frac{a_{1}}{x_{1}}, \frac{a_{k}}{x_{k}}\right) \text {. }
$$

For $j=2, \ldots, n-1$ and $k=j, j+1, \ldots, n$ set

$$
b_{j k}=A\left(\frac{b_{(j-1) j}}{a_{(j-1) j}}, \frac{b_{(j-1) k}}{a_{(j-1) k}}\right) .
$$

For our construction to work we need to choose the $x_{i}$ so that the following inequalities hold:

$$
\left|\frac{a_{i}}{x_{i}}\right|<1, \quad i=1, \ldots, n .
$$

In step 1 we chose $x_{1}$ so this holds for $i=1$.

For all $j, k$ such that $j<k$ we also need to have

$$
\left|\frac{b_{j k}}{a_{j k}}\right|<1
$$

To see that we can choose the $x_{i}$ 's so that these inequalities are all satisfied, note first that for fixed $j$, and all $k>j,\left|x_{k}\right| \rightarrow 1$ implies $\left|a_{j k}\right| \rightarrow 1$. 
Next, as $\left|x_{j}\right| \rightarrow 1$,

$$
\limsup \left|b_{1 j}\right| \leq\left|A\left(\frac{a_{1}}{x_{1}}, a_{j} e^{\theta_{j}}\right)\right|=B_{1 j}<1,
$$

where $\theta_{j}$ is chosen so that $\arg a_{j} e^{\theta_{j}}=\arg \frac{a_{1}}{x_{1}}+\pi$ and $B_{1 j}$ is maximal.

Choose $x_{1}$ so that, if the remaining $\left|x_{i}\right|$ 's are close enough to 1 , inequalities (2.4) and (2.5) hold with $j=1$.

We now find bounds

$$
\limsup _{\left|x_{j}\right| \rightarrow 1}\left|b_{2 j}\right| \leq\left|A\left(\frac{b_{12}}{a_{12}}, B_{1 j} e^{\theta_{j}}\right)\right|=B_{2 j}<1,
$$

where again $\theta_{j}$ is chosen to maximize $B_{2 j}$.

We repeat this process, choosing $x_{3}, \ldots, x_{n-1}, x_{n}$, in turn so that all the inequalities above hold.

Step 3. Define the functions $g_{k}(z): \Delta \rightarrow \Delta, k=2, \ldots, n$ recursively by

$$
A\left(x_{k}, z\right) g_{k}\left(A\left(x_{k}, z\right)\right)=A\left(\frac{b_{(k-1) k}}{a_{(k-1) k}}, g_{(k-1)}\left(A\left(x_{(k-1)}, z\right)\right)\right) .
$$

Now take $g_{n}(z)$ to be the function $g_{n}(z) \equiv 0$. Then work back through equations (2.6) to obtain the functions $g_{1}$ and $f$.

Check that $f\left(x_{i}\right)=\frac{a_{i}}{x_{i}}$ for $i=1, \ldots, n$, so that the points $x_{i}$ and the function $f$ are as required by the lemma.

Remark 2.2. In the lemma above, $n$ points $a_{1}, \ldots, a_{n}$ were given and we chose points $x_{1}, \ldots, x_{n}$ which satisfy inequalities 2.4 and 2.5. Now suppose we add one point $a_{n+1}$ to the list of the given points $a_{1}, \ldots, a_{n}\left(a_{n+1} \neq a_{i}\right.$, for $\left.1 \leq i \leq n\right)$. The first $n$ chosen points $x_{1}, \ldots, x_{n}$ don't need to change because we only need to choose $x_{n+1}$ to satisfy the following inequalities:

$$
\begin{aligned}
& \left|\frac{a_{n+1}}{x_{n+1}}\right|<1, \\
& \left|\frac{b_{j(n+1)}}{a_{j(n+1)}}\right|<1,
\end{aligned}
$$

for $j \leq n$. By an argument similar to the one that we made in the proof of the lemma above and by using the non-relative compactness of $X$ in $\Delta$, we can choose $x_{n+1}$ close enough to the boundary of the unit disk that all the inequalities above are satisfied. In the case that $n$ points $a_{1}, \ldots, a_{n}$ are given and we choose $x_{1}, \ldots, x_{n}$, the function $f$ is constructed in terms of $a_{1}, \ldots, a_{n}$ and $x_{1}, \ldots, x_{n}$. When $a_{n+1}$ is added to the list of given points and we choose one more point $x_{n+1}$, the new constructed function, say $g$, is given in terms of $a_{1}, \ldots, a_{n+1}$ and $x_{1}, \ldots, x_{n+1}$. The function $g$ is different from $f$. However, at each $x_{i}, 1 \leq i \leq n, f$ and $g$ have the same value; that is, $f\left(x_{i}\right)=g\left(x_{i}\right)=a_{i} / x_{i}$, for $1 \leq i \leq n$. In addition, $g\left(x_{n+1}\right)=a_{n+1} / x_{n+1}$.

Theorem 2.1 (The Key Theorem). Let $X$ be a non-relatively compact subdomain of $\Delta$. Let $a_{1}, a_{2}, a_{3}, \ldots$ be a sequence of infinitely many distinct points in $\Delta \backslash\{0\}$. Then there exists a holomorphic function $f: \Delta \rightarrow \Delta$ and a sequence of points $x_{1}, x_{2}, x_{3}, \ldots \in X$ such that for all $i=1,2,3, \ldots, f\left(x_{i}\right)=a_{i}$ and $f(0)=0$. 
Proof. First, we construct a sequence of functions $\left\{f_{n}\right\}_{n=1}^{\infty}$ in the following way: Consider the first point of this sequence, $a_{1}$. By Lemma 2.1, there exist a point $x_{1} \in X$ and a function $f_{1}: \Delta \rightarrow \Delta$ such that $f_{1}\left(x_{1}\right)=a_{1} / x_{1}$. Next, consider the first two points of the sequence, $a_{1}$ and $a_{2}$. By the remark above, $x_{1}$ does not need to change and there exist a point $x_{2}$ and a function $f_{2}: \Delta \rightarrow \Delta$ such that $f_{2}\left(x_{1}\right)=a_{1} / x_{1}$ and $f_{2}\left(x_{2}\right)=a_{2} / x_{2}$. In general, in order to construct $f_{n}$, we consider the first $n$ points of the sequence, $a_{1}, \ldots, a_{n}$. By the remark above, the points $x_{1}, \ldots, x_{n-1}$ that were selected for constructing $f_{n-1}$, do not need to change and there exist a point $x_{n}$ and a function $f_{n}: \Delta \rightarrow \Delta$ such that $f_{n}\left(x_{i}\right)=a_{i} / x_{i}$, for $1 \leq i \leq n$. Therefore, we have a sequence of functions $f_{1}, f_{2}, f_{3}, \ldots$ and a sequence of points $x_{1}, x_{2}, x_{3}, \ldots \in X$ such that for every fixed $n$,

$$
f_{n}\left(x_{i}\right)=a_{i} / x_{i},
$$

for $i \leq n$. That is, $f_{1}\left(x_{1}\right)=a_{1} / x_{1}$,

$f_{2}\left(x_{1}\right)=a_{1} / x_{1}, f_{2}\left(x_{2}\right)=a_{2} / x_{2}$,

$f_{3}\left(x_{1}\right)=a_{1} / x_{1}, f_{3}\left(x_{2}\right)=a_{2} / x_{2}, f_{3}\left(x_{3}\right)=a_{3} / x_{3}$,

$f_{n}\left(x_{1}\right)=a_{1} / x_{1}, f_{n}\left(x_{2}\right)=a_{2} / x_{2}, f_{n}\left(x_{3}\right)=a_{3} / x_{3}, \ldots, f_{n}\left(x_{n}\right)=a_{n} / x_{n}$.

Now, for every $n \geq 1$, define

$$
h_{n}(z)=z f_{n}(z) .
$$

It is clear that $h_{n}(0)=0$ and for every fixed $n$,

$$
h_{n}\left(x_{i}\right)=a_{i}
$$

for $i \leq n$.

The sequence $h_{1}, h_{2}, h_{3}, \ldots$ is a normal family, so by Montel's Theorem there is a subsequence which converges locally uniformly to a holomorphic map $f$ from the unit disk to the unit disk with $f(0)=0$ and $f\left(x_{i}\right)=a_{i}$ for $i=1,2,3, \ldots$.

\section{THE MAIN THEOREM}

Theorem 3.1. Suppose $X$ is a non-relatively compact subdomain of $\Delta$. Then, for any boundary point $c \in \partial X$, there is an iterated function system with $c$ as a limit function.

Proof. Let $c_{0}, c_{1}, c_{2}, c_{3}, \ldots$ be a sequence of distinct points in $X$ which converges to $c$. We claim that there exists a function $f_{1}: \Delta \rightarrow X$ and a sequence of points $c_{11}, c_{12}, c_{13}, \ldots \in X$ such that for all $i=1,2,3, \ldots, f\left(c_{1 i}\right)=c_{i}$ and $f_{1}(0)=c_{0}$.

Now, we prove this claim. Since $X$ is a hyperbolic domain, it has a holomorphic covering $g: \Delta \rightarrow X$ such that $g(0)=c_{0}$. Let $a_{1}, a_{2}, a_{3}, \ldots$ be a sequence in $\Delta$ that is mapped onto the sequence $c_{1}, c_{2}, c_{3}, \ldots$ by $g$. By Theorem 2.1, there is a function $h: \Delta \rightarrow \Delta$ and a sequence of points $c_{11}, c_{12}, c_{13}, \ldots \in X$ such that for all $i=1,2,3, \ldots, h\left(c_{1 i}\right)=a_{i}$ and $h(0)=0$. Define $f_{1}(z)=g \circ h(z)$. It is clear that $f_{1}$ is a function from the unit disk into $X$ with $f\left(c_{1 i}\right)=c_{i}$ and $f_{1}(0)=c_{0}$. This proves our claim. By the same argument there is a function $f_{2}: \Delta \rightarrow X$ and a sequence of points $c_{212}, c_{213}, c_{214}, \ldots \in X$ such that for all $i=2,3,4, \ldots, f_{2}\left(c_{21 i}\right)=c_{1 i}$ and $f_{2}(0)=c_{11}$. 
By using the same argument repeatedly, we can obtain a sequence of functions $f_{1}, f_{2}, f_{3}, \ldots$ from $\Delta$ to $X$ such that for every fixed $n$,

$$
f_{n}\left(c_{n(n-1) \ldots 21 i}\right)=c_{(n-1) \ldots 21 i},
$$

for $i=n, n+1, n+2, \ldots$ and $f_{n}(0)=c_{(n-1)(n-2) \ldots 1(n-1)}$.

Now, let's look at the backward compositions and their values at 0 :

$F_{1}(0)=f_{1}(0)=c_{0}$,

$F_{2}(0)=f_{1} \circ f_{2}(0)=c_{1}$,

$F_{3}(0)=f_{1} o f_{2} o f_{3}(0)=c_{2}$,

and in general, $F_{n}(0)=f_{1} o f_{2} o \ldots o f_{n}(0)=c_{n-1}$.

Since the sequence $c_{n}$ converges to $c$, any convergent subsequence of $F_{n}$ will also converge to $c$.

Corollary 3.1. Suppose $X$ is a non-relatively compact subdomain of $\Delta$. Then there is an iterated function system $\left\{F_{n}\right\}$ with the following property: If $p$ is a point in $\bar{X}$, it can be realized by one of the accumulation points of $\left\{F_{n}\right\}$. In other words, there is an iterated function system such that any point in $\bar{X}$ can be realized by one of its limit functions.

Proof. Let $\left\{c_{n}\right\}$ be a sequence consisting of all rational points (i.e. points with rational coordinates) in $X$. Using the same argument that we made in the proof of the previous theorem, there exists an iterated function system $\left\{F_{n}\right\}$ with the property that $F_{n}(0)=c_{n-1}$. Let $p$ be a point in $\bar{X}$. Since $\left\{c_{n}\right\}$ is dense in $\bar{X}$, there is a subsequence $\left\{c_{n_{j}}\right\}$ which converges to $p$. Now, consider the corresponding subsequence $\left\{F_{n_{j}+1}\right\}$. We have $F_{n_{j}+1}(0)=c_{n_{j}}$. Consequently, $\left\{F_{n_{j}+1}(0)\right\}$ converges to $p$. Therefore, the limit of the subsequence $\left\{F_{n_{j}+1}\right\}$, which is a limit function of $\left\{F_{n}\right\}$, realizes $p$.

\section{ACKNOWLEDGMENTS}

The author would like to thank Linda Keen for many helpful discussions. The author also thanks Nikola Lakic for several interesting conversations and is grateful to Saeed Zakeri for his useful comments.

\section{REFERENCES}

1. A. F. Beardon, T. K. Carne, D. Minda and T. W. Ng, Random iteration of analytic maps, Ergodic Theory and Dynam. Sys. 24 (2004), 659-675. MR2060992 (2005b:37086)

2. L. Carleson and T. W. Gamelin, Complex Dynamics, Springer-Verlag, 1993. MR:1230383 (94h:30033)

3. J. Gill, Compositions of analytic functions of the form $F_{n}(z)=F_{n-1}\left(f_{n}(z)\right), f_{n}(z) \rightarrow f(z)$, J. Comput. Appl. Math. 23 (2) (1988), 179-184. MR959476 (89i:30028)

4. L. Keen and N. Lakic, Accumulation points of iterated function systems, in Complex Dynamics: Twenty-Five Years after the Appearance of the Mandelbrot Set, Contemp. Math., 396, eds. R. Devaney and L. Keen, Amer. Math. Soc., 2006. MR2209089 (2007e:30030)

5. L. Keen and N. Lakic, Random holomorphic iterations and degenerate subdomains of the unit disk, Proc. Amer. Math. Soc. 134 (2006), no. 2, 371-378 (electronic). MR 2176004 (2007a:37056)

6. L. Keen and N. Lakic, Hyperbolic Geometry from a Local Viewpoint, Cambridge University Press, 2007. MR2354879 (2008h:30001) 
7. L. Lorentzen, Compositions of contractions, J. Comput. Appl. Math. 32 (1990), 169-178. MR 1091787 (92f:30035)

8. J. Milnor, Dynamics in One Complex Variable, 3rd edition, Princeton University Press, 2006. MR2193309 (2006g:37070)

Department of Mathematics, The City University of New York Graduate Center, 365 Fifth Avenue, New York, New York 10016

Current address: Department of Mathematics, Fordham University, 441 E. Fordham Road, Bronx, New York 10458

E-mail address: tavakoli@fordham.edu 